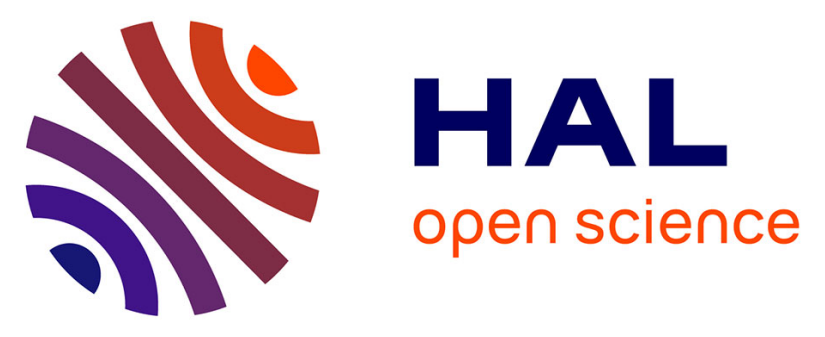

\title{
Influence of various process conditions on surface finishes induced by the direct metal deposition laser technique on a Ti-6Al-4V alloy
}

Myriam Gharbi, Patrice Peyre, Cyril Gorny, Muriel Carin, Simon Morville, Philippe Le Masson, Denis Carron, Rémy Fabbro

\section{To cite this version:}

Myriam Gharbi, Patrice Peyre, Cyril Gorny, Muriel Carin, Simon Morville, et al.. Influence of various process conditions on surface finishes induced by the direct metal deposition laser technique on a Ti-6Al-4V alloy. Journal of Materials Processing Technology, 2012, 213, pp.791-800. 10.1016/j.jmatprotec.2012.11.015 . hal-00987226

\section{HAL Id: hal-00987226 https://hal.science/hal-00987226}

Submitted on 6 May 2014

HAL is a multi-disciplinary open access archive for the deposit and dissemination of scientific research documents, whether they are published or not. The documents may come from teaching and research institutions in France or abroad, or from public or private research centers.
L'archive ouverte pluridisciplinaire HAL, est destinée au dépôt et à la diffusion de documents scientifiques de niveau recherche, publiés ou non, émanant des établissements d'enseignement et de recherche français ou étrangers, des laboratoires publics ou privés. 


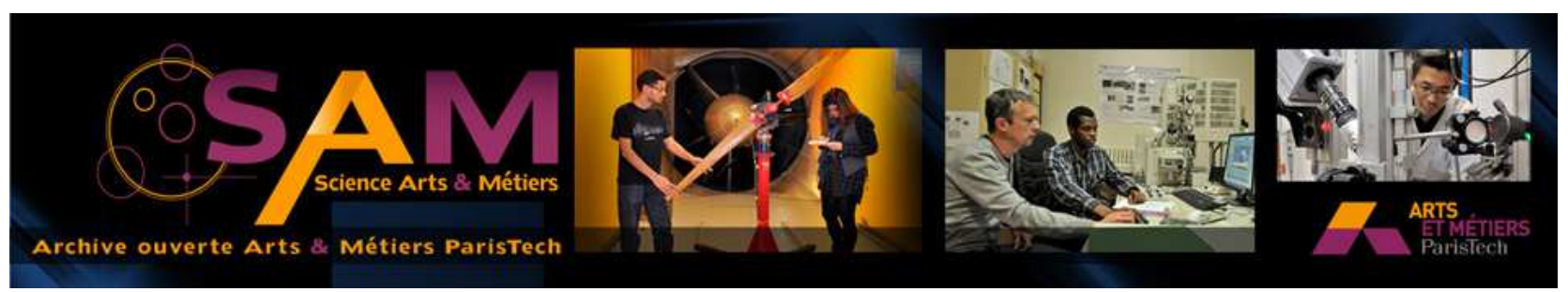

\section{Science Arts \& Métiers (SAM)}

is an open access repository that collects the work of Arts et Métiers ParisTech researchers and makes it freely available over the web where possible.

This is an author-deposited version published in: http://sam.ensam.eu

Handle ID: .http://hdl.handle.net/10985/8099

\section{To cite this version :}

Myriam GHARBI, Patrice PEYRE, Cyril GORNY, Muriel CARIN, Simon MORVILLE, Philippe LE MASSON, Denis CARRON, Rémy FABBRO - Influence of various process conditions on surface finishes induced by the direct metal deposition laser technique on a Ti-6Al-4V alloy - Journal of Materials Processing Technology - Vol. 213, p.791-800. - 2012 


\title{
Influence of various process conditions on surface finishes induced by the direct metal deposition laser technique on a Ti-6Al-4V alloy
}

\author{
Myriam Gharbi $^{\mathrm{a}}$, Patrice Peyre ${ }^{\mathrm{a}, *}$, Cyril Gorny $^{\mathrm{a}}$, Muriel Carin $^{\mathrm{b}}$, Simon Morville $^{\mathrm{b}}$, \\ Philippe Le Masson ${ }^{\mathrm{b}}$, Denis Carron ${ }^{\mathrm{b}}$, Rémy Fabbro ${ }^{\mathrm{a}}$ \\ a PIMM, UMR 8006 CNRS-Arts et métiers ParisTech, 75013 Paris, France \\ ${ }^{\mathrm{b}}$ LIMATB, Université de Bretagne-Sud, 56321 Lorient, France
}

\section{Keywords:}

Laser

Manufacturing

Deposition

Surface

Titanium
The direct metal deposition (DMD) with laser is a free-form metal deposition process for manufacturing dense pieces, which allows generating a prototype or small series of near net-shape structures. One of the most critical issues is that produced pieces have a deleterious surface finish which systematically requires post machining steps. This problem has never been fully addressed before.

The present work describes investigations on the DMD process, using an Yb-YAG disk laser, and a widely used titanium alloy (Ti-6Al-4V) to understand the influence of the main process parameters on the surface finish quality. The focus of our work was: (1) to understand the physical mechanisms responsible for deleterious surface finishes, (2) to propose different experimental solutions for improving surface finish.

In order to understand the physical mechanisms responsible for deleterious surface finishes, we have carried out: (1) a precise characterization of the laser beam and the powder stream; (2) a large number of multi-layered walls using different process parameters $\left(P(\mathrm{~W}), V(\mathrm{~m} / \mathrm{min}), D_{\mathrm{m}}(\mathrm{g} / \mathrm{min})\right.$, Gaussian or uniform beam distribution); (3) a real time fast camera analysis of melt pool dynamics and melt-pool - powder stream coupling; (4) a characterization of wall morphologies versus process parameters using 2D and 3D profilometry.

The results confirm that surface degradation depends on two distinct aspects: the sticking of nonmelted or partially melted particles on the free surfaces, and the formation of menisci with more or less pronounced curvature radii. Among other aspects, a reduction of layer thickness and an increase of melt-pool volumes to favor re-melting processes are shown to have a beneficial effect on roughness parameters. Last, a simple analytical model was proposed to correlate melt-pool geometries to resulting surface finishes.

\section{Introduction}

The direct metal deposition (DMD) laser technique is a recentlydeveloped manufacturing technique that allows obtaining drafts of complex metallic parts from a three dimensional CAD model as reminded by Pinkerton (2010), even if technical issues still exist (stability of the DMD, process control, geometrical limits) before a widespread industrialization. The process uses a high power laser (usually Nd:YAG, fiber or diode laser) focused onto a metallic substrate to generate a molten pool, where a coaxial powder feeding increases the material volume and contributes to the formation of a solid layer. The substrate is then scanned on $(x, y, z)$ directions relative to the laser + powder nozzle head and the layer-by-layer

\footnotetext{
* Corresponding author.

E-mail address: patrice.peyre@ensam.eu (P. Peyre).
}

additive manufacturing of complex 3D shapes becomes possible. Many materials, including graded materials, have already been more or less successfully attempted, with a specific focus on aeronautical materials. For instance, considering the specific case of Ti-6Al-4V titanium alloy, Maisonneuve et al. (2007) or Bontha (2006) pointed out the dependence between process parameters and grain structure (columnar or equiaxed), whereas Brandl et al. (2011) demonstrated that the use of a wire instead of powder could lead to good-yet anisotropic mechanical properties. From the previously mentioned publications and many other ones, it appears that, with a DMD laser technique, and whatever the projected material, similar or superior mechanical properties than foundry techniques can be achieved, either in static condition or under fatigue loading.

Among the various factors involved in the DMD process, one can distinguish between 1st order factors: Laser power $P_{0}(\mathrm{~W})$, scan speed $V_{0}(\mathrm{~m} / \mathrm{min})$, laser diameter $d_{0}(\mathrm{~mm})$, and mass feed rate $D_{\mathrm{m}}^{0}$ 
(g/min), and 2nd order factors: defocussing distance, gas shielding nature and velocity, time pause between passes, spatial distributions of laser and powder etc. ... All these factors influence the thermal history $T=f(x, y, z, t)$ of the part, and contribute not only to the melt-pool shapes, and the resulting layer growth, but also to the final metallurgical and mechanical properties

Recently (2006-2011), intensive numerical work has been carried out to model the DMD process, starting from the laser-powder interaction, to the thermo-mechanical calculation of residual stresses, including or-not metallurgical aspects. Authors like Fathi et al. (2006) proposed rather simplified predictive models to predict the geometrical characteristics of the walls by assuming a solid state during the process. Whereas, more complex thermohydraulic calculations considered free moving surfaces, either on 2D multilayers configurations like Morville et al. (2011), or on 3D single layer as shown by Qi et al. (2006) or Kumar and Roy (2009). Such numerical approaches allowed calculating wall dimensions, fluid flow and realistic temperature distribution inside the clad layers.

In turn, the DMD process is now globally well understood even if limitations still remain such as:

1) the need of a robust and reliable process control to maintain constant local thermal conditions, and a stable and constant layer growth;

2) the occurrence of a deleterious surface finish (usually $R_{\mathrm{a}}>15 \mu \mathrm{m}$ as shown by Maisonneuve et al. (2007) on Ti-6Al-4V or Pinkerton and Li (2003) on AISI 316L), that requires intensive post-machining steps, and restraints the applications of DMD to the drafts of final parts.

A common thought is that optimum surface finishes are mostly expected when using thin and stable DMD layers, without more precisions concerning process parameters, and melt-pool shapes and dynamics. Moreover, as reminded by Pinkerton (2010), one can assume that melt-pool stability depends on various internal forces (Marangoni flow, gravitational forces) or external forces (gas pressure, recoil forces near vaporization point, dynamic forces applied by the projected powder grains) applied on the molten metal. For instance, negative thermo-capillary coefficients $d \sigma / d T$ $(\mathrm{N} / \mathrm{m} \mathrm{K})$ are expected to provoke centrifugal Marangoni fluid flow directed away from the melt-pool center. This flow could act as a shaping contribution to the melt-pool and modify the resulting

(a)

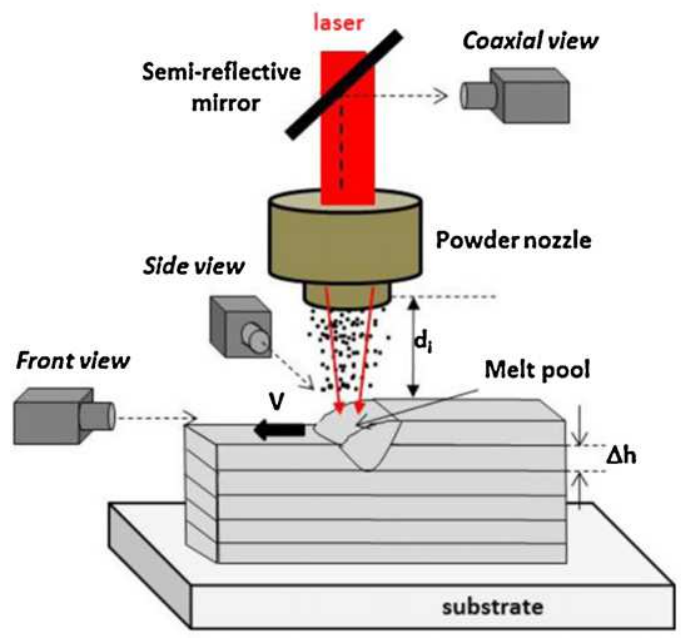

surface finish, during layer additive processes, even if such an effect has to be confirmed experimentally.

Recent work has considered surface quality as an important factor to address in itself to improve the process. For instance (Zhu et al., 2012) has shown the benefit versus surface quality of positioning the powder focus below the melt-pool, and the laser focus above. A melt-pool enlargement was shown to be the main contributor to optimum surface smoothness. It was also shown by (Alimardani et al., 2012) that an increase of the scanning speed and a real-time control of the melt-pool dimension and temperature could significantly improve surface finish.

In this context, it seemed important to understand the specific contributions of various processing parameters to surface finish, considering simultaneously the variation of melt-pool characteristics, with the final objective of reaching optimum surface finishes, and limiting post-machining steps. Considering a widely investigated Ti-6Al-4V titanium alloy, and a large range of process parameters (laser power $P_{0}(\mathrm{~W})$, scan speed $V(\mathrm{~m} / \mathrm{min})$, mass feed rate $D_{\mathrm{m}}(\mathrm{g} / \mathrm{min})$, laser-powder interaction distance $d_{\mathrm{i}}(\mathrm{mm})$, beam distribution (Gaussian or top-hat), gas shielding conditions), this paper addresses surface finish variations, and aims at correlating them with melt-pool shapes and dimensions.

\section{Experimental conditions}

\subsection{Direct metal deposition conditions}

DMD tests have been carried out using a HL 10,002 continuous wave disk Yb:YAG laser operating at $1.03 \mu \mathrm{m}$, with $320-700 \mathrm{~W} \mathrm{cW}$ laser powers. Two configurations were used:

(1) a $1.3 \mathrm{~mm}$ diameter defocused Gaussian laser spot provided by a $0.4 \mathrm{~mm}$ optical fiber delivery, and respectively $200 \mathrm{~mm}$ collimation and focusing lens in the laser head. The laser beam was used with a $+5 \mathrm{~mm}$ defocusing condition, resulting in a $1.3 \mathrm{~mm}$ diameter beam on the substrate;

(2) a $1.68 \mathrm{~mm}$ diameter top-hat laser spot obtained with a $600 \mu \mathrm{m}$ optical fiber, a $100 \mathrm{~mm}$ collimating lens and a $280 \mathrm{~mm}$ focusing lens.

For the powder distribution, a helicoïdal powder delivery nozzle was used, where the Titanium powder (45-75 $\mu \mathrm{m}$, TLS Technik) is delivered coaxially with the laser beam, resulting in a $d_{\mathrm{p}} \approx 3 \mathrm{~mm}$ powder focus diameter, and with average mass feed rates $D_{\mathrm{m}}$ in (b)

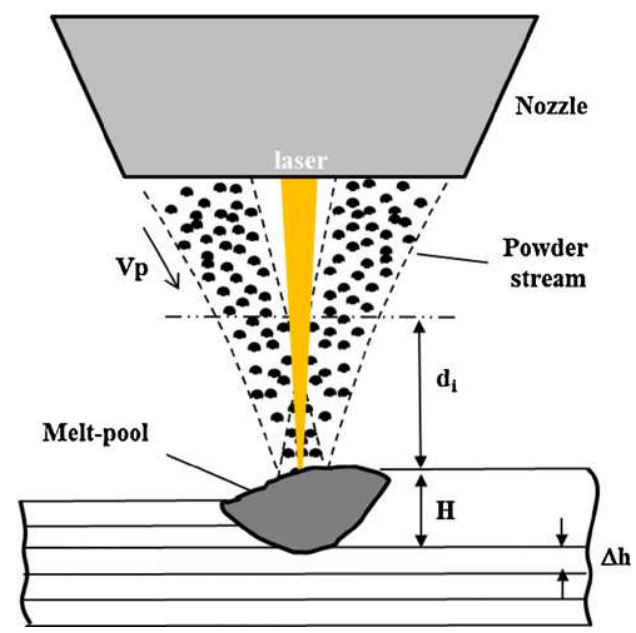

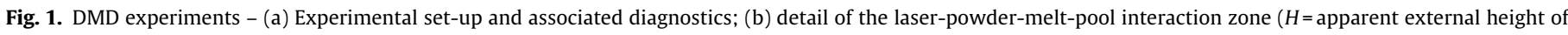
the melt-pool, $\Delta h=$ additive layer height). 
(a)

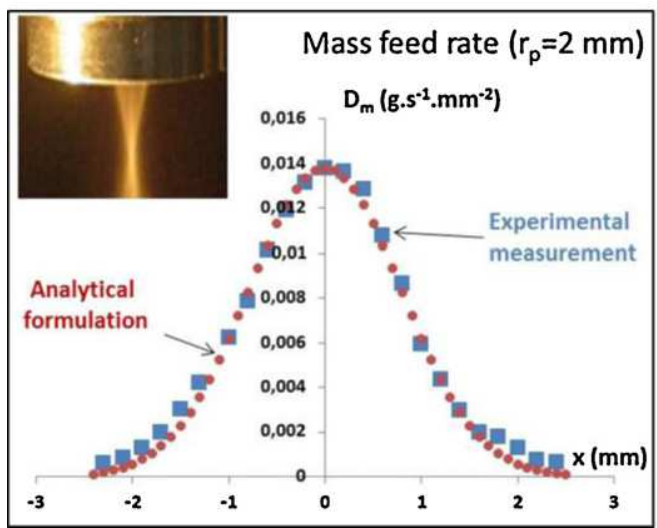

(b)

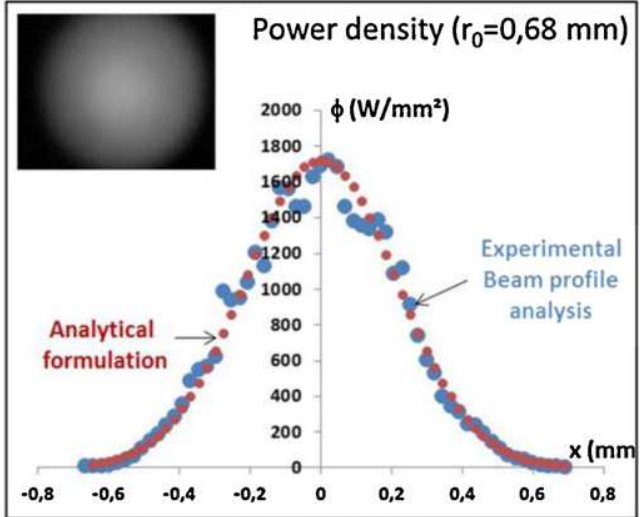

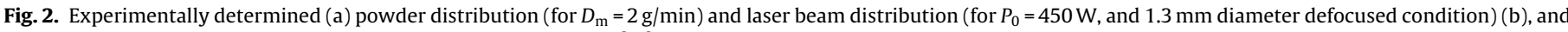
associated near-Gaussian analytical formulations $\left(f(x)=A\right.$. exp $\left.\left(-5 x^{2} / r^{2}\right)\right)$.

the $1 \mathrm{~g} / \mathrm{min}$ to $3 \mathrm{~g} / \mathrm{min}$ regime. The relative positioning of laser beam versus powder stream is presented in Fig. 1a $\left(d_{\mathrm{i}}\right.$ : interaction distance between powder stream and laser beam). Argon was used as a driving and shielding gas, in order to ensure powder transportation, and to limit oxidation phenomena. All the DMD tests have been carried out with $40 \mathrm{~mm}$-length/6-15 mm-height walls, starting from $2 \mathrm{~mm}$-thick titanium sheets (Fig. 1b), and with scan speeds in the $0.1 \mathrm{~m} / \mathrm{min}$ to $0.6 \mathrm{~m} / \mathrm{min}$ range. Experimental conditions are summarized in Table 1. Last, it has to be mentioned that most of the tests were carried out with a local argon shielding (through the nozzle), whereas a few additional tests were carried out on a Lens Optomec-450 industrial set-up, with a controlled $\mathrm{O}_{2}$ level of $20 \mathrm{ppm}$ in the whole cell.

The experimental determination of mass feed rate distribution versus $x$-axis was carried out using a specific device where a metallic sheet with a $0.3 \mathrm{~mm}$ diameter laser-drilled hole is moved below the powder nozzle, and allows determining the local mass feed rate at any location of the powder stream. The laser beam profile was analyzed by an industrial beam analyzer. The $1.3 \mathrm{~mm}$ diameter laser spot, and powder distributions were both shown to be quasi-Gaussian in shape, as shown in Fig. 2.

In turn, coincidently, similar analytical formulations could be used for the two spatial distributions (Eqs. (1) and (2)).

$D\left(\mathrm{~g} \cdot \mathrm{s}^{-1} \cdot \mathrm{m}^{-2}\right)=5 \cdot \frac{D_{\mathrm{m}}}{\left(\pi r_{\mathrm{p}}^{2}\right)} \cdot \exp \left(-\frac{5 x^{2}}{r_{\mathrm{p}}^{2}}\right)$

with $r_{\mathrm{p}}=$ powder stream radius $(=2 \mathrm{~mm}), v_{\mathrm{p}}=$ powder velocity $(\mathrm{m} / \mathrm{s}), d_{\mathrm{i}}=$ interaction distance $(\mathrm{mm}), D_{\mathrm{m}}=$ average mass feed rate $(\mathrm{g} / \mathrm{s})$

$\varnothing\left(\mathrm{W} \cdot \mathrm{m}^{-2}\right)=5 \frac{P_{0}}{\pi r_{\text {laser }}^{2}} \cdot \exp \left(-\frac{5 x^{2}}{r_{\text {laser }}^{2}}\right)$

with $r_{\text {laser }}=$ laser beam radius $(=0.65 \mathrm{~mm}), P_{0}=$ laser power $(\mathrm{W})$

On the other hand, additional DMD tests were carried out with a "top-hat" uniform distribution $\left(f=P_{0} /\left(\pi r_{\text {laser }}^{2}\right)\right.$ ), and a $1.68 \mathrm{~mm}$ diameter, in order to estimate the influence of beam distribution on the melt-pool shape, and surface finish.

Considering the laser-powder interaction during the time-offlight (interaction time $t_{0}=d_{\mathrm{i}} / v_{\mathrm{p}}$ ) of a powder grain, an estimated

Table 1

Experimental DMD conditions.

\begin{tabular}{lllll}
\hline Parameters & $d_{\text {laser }}(\mathrm{mm})$ & $P(\mathrm{~W})$ & $V(\mathrm{~m} / \mathrm{min})$ & $D_{\mathrm{m}}(\mathrm{g} / \mathrm{min})$ \\
\hline Values & 1.3 & $320,400,500$ & $0.1,0.2,0.4,0.6$ & $1,2,3$ \\
\hline
\end{tabular}

calculation of powder temperature increase $\Delta T$ could be made using a recent and simplified model by Qi et al. (2006) where the absorption of laser light by powder was adjusted by an absorption coefficient " $A$ " ( $\approx 0.4$ on titanium) (Eq. (3)). In this model, interactions between particles and particle/gas thermal losses were neglected. If we only consider the $1.3 \mathrm{~mm}$ diameter Gaussian laser spot, the two distinct interaction distances $d_{\mathrm{i}}$ used during our tests $(1 \mathrm{~mm}$ and $3 \mathrm{~mm}$ ), correspond either to a powder mostly maintained at a solid state, or to a powder that is molten before contacting the melt-pool (Fig. 3).

$\Delta T=\frac{3 A \phi(x, y)}{4 r_{\mathrm{g}} \rho C_{\mathrm{p}}} \cdot \frac{d_{\mathrm{i}}}{v_{\mathrm{p}}}$

with $r_{\mathrm{g}}=$ powder grain radius $(\mathrm{mm}), \quad v_{\mathrm{p}}=$ powder velocity $(\approx 1.5 \mathrm{~m} / \mathrm{s}), d_{\mathrm{i}}=$ interaction distance $(\mathrm{mm})$.

\subsection{Real-time diagnostics}

A simultaneous coaxial and lateral recording of melt-pool sizes and dynamics was carried out, using two synchronized fast cameras with C-Mos sensors (Photron), at frequency rates up to $5000 \mathrm{~Hz}$, using KG3 filters to cut the laser wavelength $(1.053 \mu \mathrm{m})$, and halogen lights to improve the contrast of recorded images. This allowed us to: (1) investigate powder stream (projected powder velocity $v_{\mathrm{p}}$ ),(2) investigate powder/melt-pool interaction, (3) analyze meltpool flow (powder velocity in the melt $v_{\mathrm{p}}$, fluid velocity $v_{\mathrm{f}}$ when

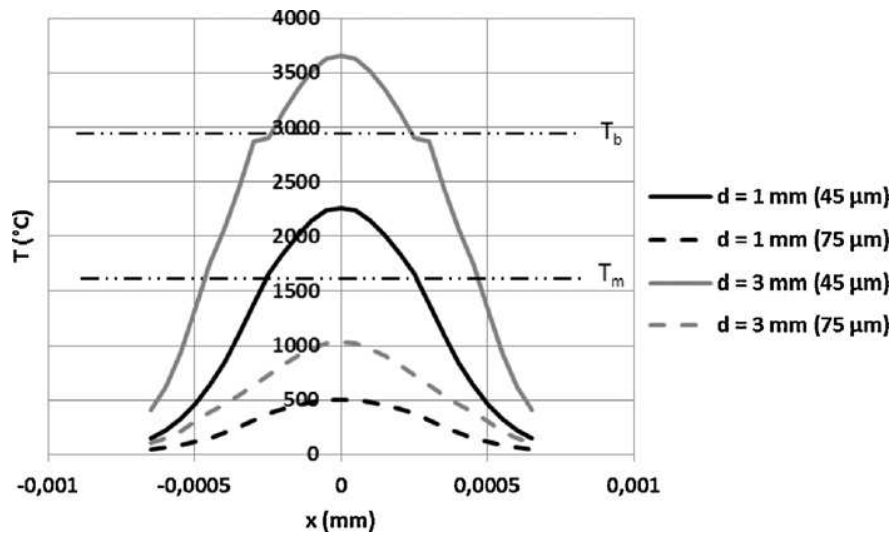

Fig. 3. Temperature distribution $T=f(x)$ inside the powder stream for 2 different interaction distances and powder diameters (based upon the analytical model by Qi et al. (2006), for $P_{0}=500 \mathrm{~W}$. 
(a)

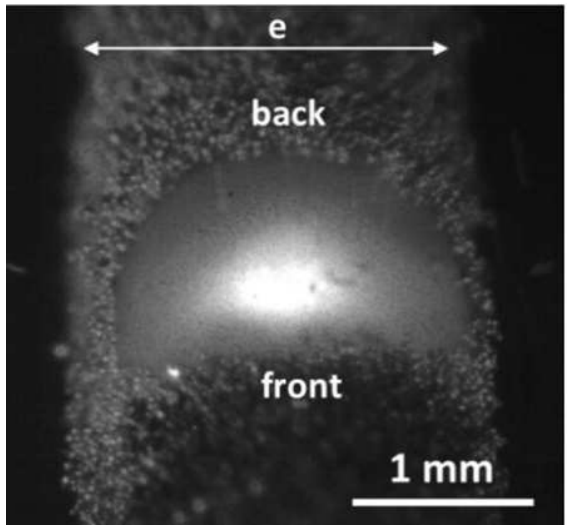

(b)

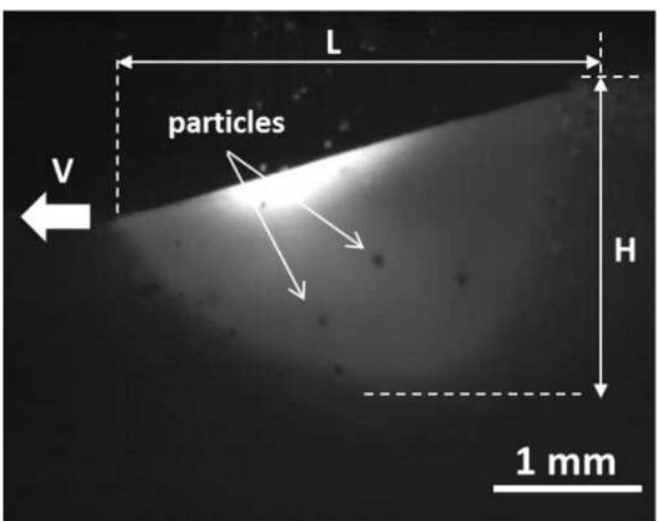

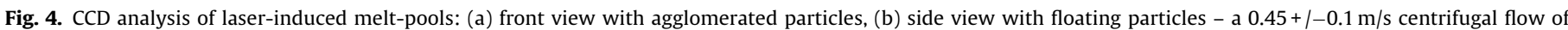
particles in the melt-pool is estimated, whatever the process conditions.

Table 2

Thermo-physical properties of Ti-6Al-4V (A: absorptivity; S: solid; L: liquid).

\begin{tabular}{|c|c|c|c|c|c|c|c|c|}
\hline$\rho(\mathrm{S} / \mathrm{L})\left[\mathrm{kg} / \mathrm{m}^{3}\right]$ & $\mathrm{k}(\mathrm{S} / \mathrm{L})\left[\mathrm{W} \mathrm{m}^{-1} \mathrm{~K}^{-1}\right]$ & $C_{\mathrm{p}}(\mathrm{S} / \mathrm{L})\left[\mathrm{J} \mathrm{kg}^{-1} \mathrm{~K}^{-1}\right]$ & $\eta[\mathrm{Pas}]$ & $L_{\mathrm{f}}\left[\mathrm{J} \mathrm{kg}^{-1}\right]$ & $A$ & $T_{\mathrm{m}}[\mathrm{K}]$ & $\sigma[\mathrm{N} / \mathrm{m}]$ & $\gamma=d \sigma / d T\left[\mathrm{~N} \mathrm{~m}^{-1} \mathrm{~K}^{-1}\right]$ \\
\hline $4400 / 4200$ & $20 / 35$ & $600 / 700$ & $4.10^{-3}$ & $3.10^{5}$ & 0.4 & 1920 & 1.5 & $-2.10^{-4}$ \\
\hline
\end{tabular}

distinct, (4) quantify melt-pool dimensions (length $L$, height $H$, area $S)$ and try to correlate them with surface finish data.

\subsection{Surface analysis}

DMD surfaces exhibit a periodic structure composed of an accumulation of lateral menisci directly due to the equilibrium shapes of the melt-pool. Manufactured walls were analyzed using: (1) low and medium magnification optical microscopy to visualize the global surface aspect and the grain microstructure (after Kroll's etching), (2) scanning electron microscopy (SEM-FEG Hitachi 4802 II) to provide a local description of DMD surfaces, (3) 2D and 3D roughness analysis (Veeco Dektak 150 stylus profiler) to quantify surface topography. The surface analysis have been carried out using a $4 \mathrm{~mm}$ scanning length perpendicular to the main DMD direction with a $2.5 \mu \mathrm{m}$ tip stylus, and a $0.1 \mathrm{~mm} / \mathrm{s}$ scanning speed.

\section{Experimental DMD tests}

\subsection{Melt-pool dynamics and melt-pool dimensions versus process parameters}

When powder particles reach the molten pool, they follow two options: (1) they ricochet on the surface layers partially covered with Ti oxide, below a threshold collision angle $\theta_{\text {th }}$; (2) they enter more or less partially the melt-pool, and are transported by the fluid flow where, after full melting, they contribute to layer growth.

Powder grains contacting the melt-pool without ricocheting are transported by a Marangoni centrifugal fluid flow at a $V_{\mathrm{f}}$ velocity $\approx 0.45 \mathrm{~m} / \mathrm{s}$ estimated with fast camera (Fig. 4 ). The direction of Marangoni flow is in good agreement with a negative thermocapillary coefficient $d \sigma / d T(\mathrm{~N} / \mathrm{mK})$, which is mostly expected in molten titanium (Table 2 ).

The ratio of particles contacting the melt-pool, but not contributing to layer growth was checked analytically, by calculating the theoretical catch efficiency ratio $\vartheta$, i.e. the integral value of the $D=f(x, y)$ function (Eq. (1)) restricted by the limits of the melt-pool i.e. approximately the melt-pool width e (Eq. (4)). The comparison of the analytical prediction, and the experimental mass efficiency (=mass really incorporated in the wall/mass projected) indicates (Fig. 5) 15\% higher analytically calculated catch efficiency data. This reveals that approximately $85 \%$ of the incident particles projected inside the contours of the melt-pool are really melted whereas, the $15 \%$ remaining are attributed to particle skipping that tend to ricochet on the melt-pool surface, to particles that are shifted when interacting with other particles, or to particles that do not fully melt and stick on the surface. This skipping phenomenon is promoted by small incidence angles at the edges of the semi-hemispherical melt-pool, and large fluid velocities in the melt-pool.

$\vartheta=\frac{\int_{-\theta / 2}^{\theta / 2} D^{0} \cdot \exp \left(-5 x^{2} / r_{p}^{2}\right) \cdot d x}{\int_{-\infty}^{\infty} D^{0} \cdot \exp \left(-5 x^{2} / r_{p}^{2}\right) \cdot d x}$

with $r_{\mathrm{p}}=$ powder stream radius ( $\left.\mathrm{mm}\right), e=$ melt-pool width $(\mathrm{mm})$, $\left(D^{0}=5 D_{\mathrm{m}} / \pi r_{\mathrm{p}}^{2}\right)$.

If we now consider the variation of wall dimensions versus $\left(P_{0}\right.$, $\left.V, D_{\mathrm{m}}\right)$ process parameters, rather classical results are obtained (Fig. 6):

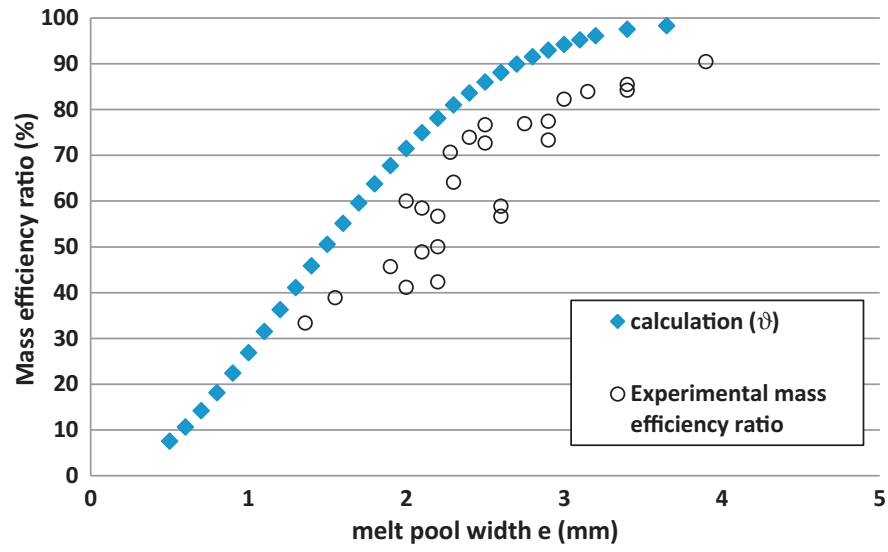

Fig. 5. Comparison between the analytical calculation of the catch efficiency mass ratio $\vartheta$ s and experimentally determined data: $15 \%$ of the powder projected into the melt-pool does not contribute to layer growth due to ricocheting or non-sticking phenomena. 
(a)

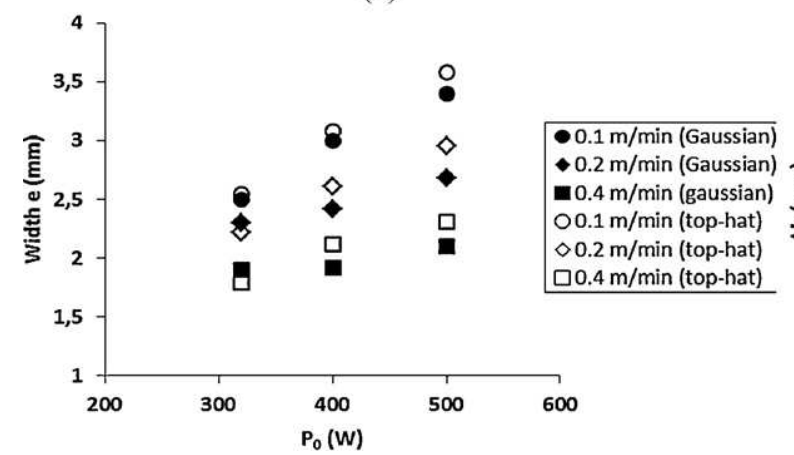

(b)

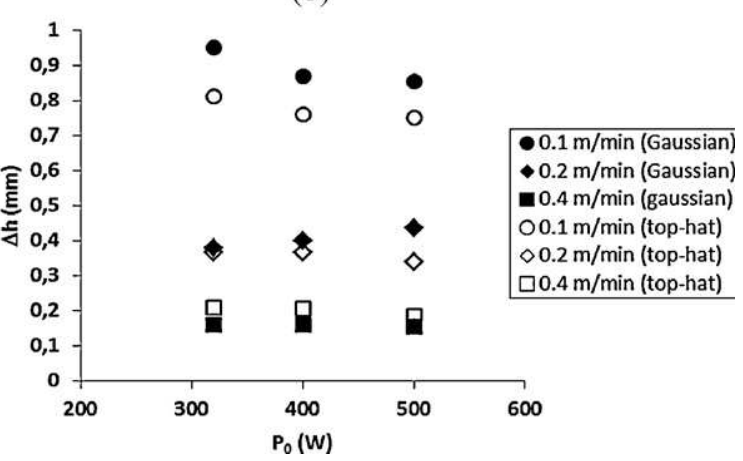

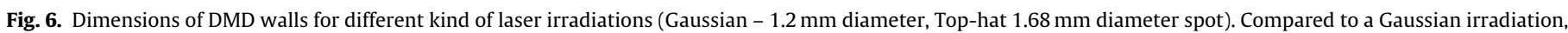
the use of a $1.68 \mathrm{~mm}$ top-hat irradiation tends to induce a moderate increase of layer widths e, and a tendency to decrease of layer heights $\Delta h$.

- Wall thicknesses increase with $P_{0}$ and $1 / V$, due to an increase of the lineic energy $P / V(\mathrm{~J} / \mathrm{m})$, but vary to a little extent with $D_{\mathrm{m}}$ as the laser beam filtering is limited in the $1-3 \mathrm{~g} / \mathrm{min}$ mass feeding range

- Layer heights $\Delta h$ increase with $D_{\mathrm{m}}$ and $1 / V$, but do not vary with $P_{0}$.

Those results can be discussed considering a recentyet-simple analytical formulation of layer height by Peyre et al. (2008) $\Delta h=D_{\mathrm{m}}^{*} \cdot L / \rho \cdot V\left(\right.$ with $\left.D_{\mathrm{m}}^{*}\left(\mathrm{~g} \mathrm{~s}^{-1} \mathrm{~m}^{-2}\right)\right)=$ average surface mass feed rate. However, such a formulation has to be modified to explain why $\Delta h$ remains constant with increasing $P_{0}$ values. For this purpose, one has to take into account the notion of efficient mass feed rate: as mass feed rate distribution is Gaussian in shape (Fig. 2a), the average mass feed rate really contributing to layer growth depends on melt-pool dimensions (length $L$ and width $e$ ). More precisely, small melt-pools will be fed by higher average feed rates (the upper part of the Gaussian distribution) that larger ones. So, an increase of layer heights $\Delta h$ with laser power $P_{0}$ will be counterbalanced by a decrease of the efficient mass feeding $D_{\mathrm{m}}^{*}$ eff with the melt-pool average size $(L+e) / 2$ (Eq. (5)).

$D_{\mathrm{m}}^{*, \mathrm{eff}}=\frac{2}{(L+e)} \cdot \int_{-(L+e) / 2}^{(L+e) / 2} D_{\mathrm{m}}^{*} \cdot d x$

$D_{\mathrm{m}}^{*}\left(\mathrm{~kg} \mathrm{~s}^{-1} \mathrm{~m}^{-2}\right)=$ average mass feed rate $=D_{\mathrm{m}} /\left(\pi \cdot r_{\mathrm{p}}^{2}\right)$,

$(\mathrm{m})=$ melt-pool length, $e(\mathrm{~m})=$ melt-pool width .

The use of a uniform top-hat distribution instead of a Gaussian beam (Fig. 6) is shown to have a rather limited effect on wall dimensions, despite a reduction of thermal gradients in the melt-pool as demonstrated recently by Gharbi et al. (2012), and a reduction of resulting centrifugal Marangoni flow. Results indicate approximately a $5 \%$ increase of wall widths "e". To understand why wall widths are nearly identical, two counterbalancing effects can be assumed: on the one hand, without considering Marangoni flow, a $1.68 \mathrm{~mm}$ top-hat heating is expected to induce wider fusion isotherms than a $1.3 \mathrm{~mm}$ Gaussian heating, but on the other hand, the reduction of centrifugal Marangoni effects tends to limit meltpool lateral expansion.

If we now consider layer heights $\Delta h$, a tendency to decrease is obtained with a uniform laser irradiation, especially at low scan speeds. This could be attributed to longer and wider melt-pool lengths, that tend to reduce the efficient mass feed rate $D_{\mathrm{m}}^{*}$, eff .

\subsection{Evolution of surface finishes with process parameters for a Gaussian beam distribution}

\subsubsection{General approach}

Using SEM analysis (Fig. 7a), two distinct contributions to surface roughening were evidenced:

(1) a microscopic contribution coming from (a) particle agglomerations mostly located in inter-layers areas and (b) solidification lines, favored by small melt-pools, and rather low temperature distributions near the melt-pool;

(2) a more macroscopic contribution: the formation of periodic menisci, directly associated with the melt-pool stability (Fig. 7a).

For differentiating macroscopic waviness contribution $\left(W_{\mathrm{a}}, W_{\mathrm{t}}\right)$ from microscopic roughness $\left(R_{\mathrm{a}}, R_{\mathrm{t}}\right)$ parameters, a cut-off filter (threshold $d_{\mathrm{th}}=80 \mu \mathrm{m}$ ) was used (Fig. 7b). The choice of a $80 \mu \mathrm{m}$ filter was justified by the maximum diameter of particles $(75 \mu \mathrm{m})$ that usually provoke micro-roughening when agglomerating on the wall edges. The $R_{\mathrm{a}}$ and $W_{\mathrm{a}}$ values correspond to arithmetic average values (Eq. (6)) whereas $R_{\mathrm{t}}$ and $W_{\mathrm{t}}$ correspond to peak-to-valley values. All measurements were carried out on a $4 \mathrm{~mm}$-length distance, using 3 to 4 tests for each DMD condition to provide us with statistically reliable data.

$R_{\mathrm{a}}, \quad W_{\mathrm{a}}=\frac{1}{d_{\mathrm{th}}} \int_{0}^{d_{\mathrm{th}}}|Z(x)| d x$

\subsubsection{Influence of $\left(P, V, D_{m}\right)$ process parameters}

3D profiles of surface finish obtained with a Gaussian beam distribution are shown in Fig. 8. For constant mass feed rate and scan speed values, the benefit of using high laser powers and thin additive layers (high scan speeds) is clearly shown. Similarly, on cross-sections (Figs. 9 and 10), the periodic menisci are clearly evidenced, together with melt-pool limits, and layer heights $\Delta h$. The top region of the samples includes the last fusion zone, and the last heat-affected zone where the local material temperature has exceeded the $\beta$ transus temperature at $910^{\circ} \mathrm{C}$ (Fig. 9) as already indicated by Maisonneuve et al. (2007) and Fachinetti et al. (2010). This $\alpha / \beta$ metallurgical transformation induces successive transition black lines on cross-sections that allow distinguishing layer heights.

An interesting point to notice about cross-sections analysis is the evolution of the melt-pool shape with laser power: for a similar additive layer thickness $(0.15 \mathrm{~mm})$ a higher laser power $(500 \mathrm{~W}$ versus $320 \mathrm{~W}$ ) promotes a more pronounced internal concavity (quantified by $\theta_{2}$ angle in Fig. 9) in the lower part of the melt-pool. This phenomenon is clearly due to an increase of lateral thermal 

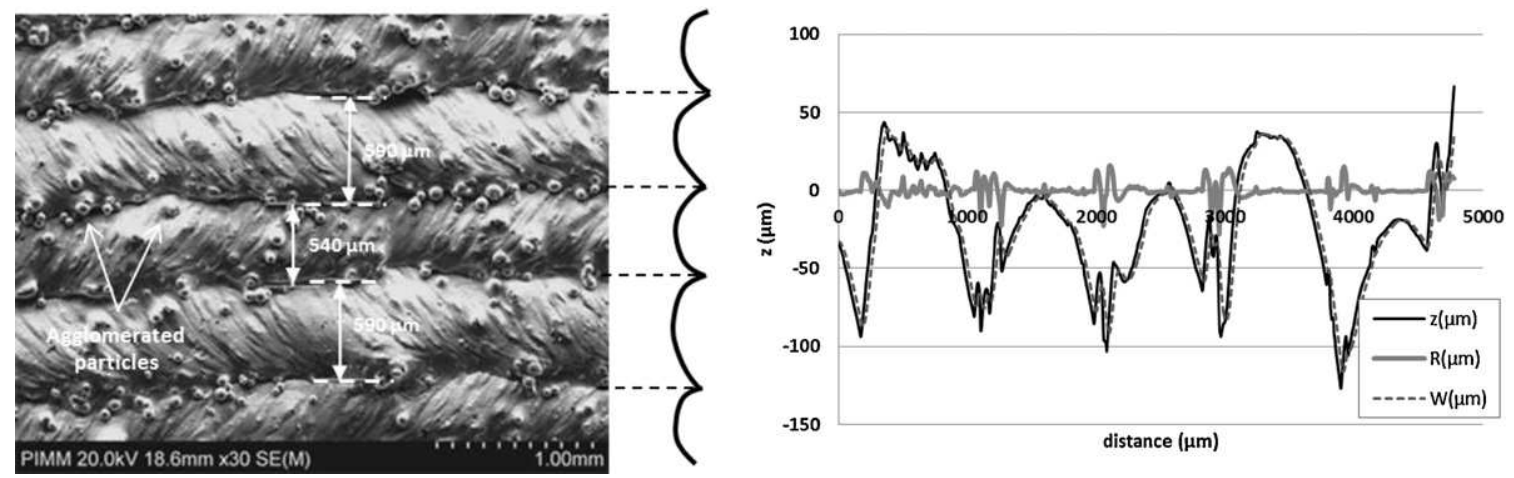

Fig. 7. (a) SEM picture of a DMD surface $(550 \mu \mathrm{m}$-height menisci, agglomerated particles accumulated in the inter-layers zones and solidification lines) $320 \mathrm{~W}-2 \mathrm{~g} / \mathrm{min}$ - $0.4 \mathrm{~m} / \mathrm{min}$, (b) 2D profile - Distinction between micro-roughness $R$ and macro-waviness $W$ using a cut-off filter of $80 \mu \mathrm{m}$ from a starting value $z$ (400 W - $0.2 \mathrm{~m} / \mathrm{min}$ $1 \mathrm{~g} / \mathrm{min})$.
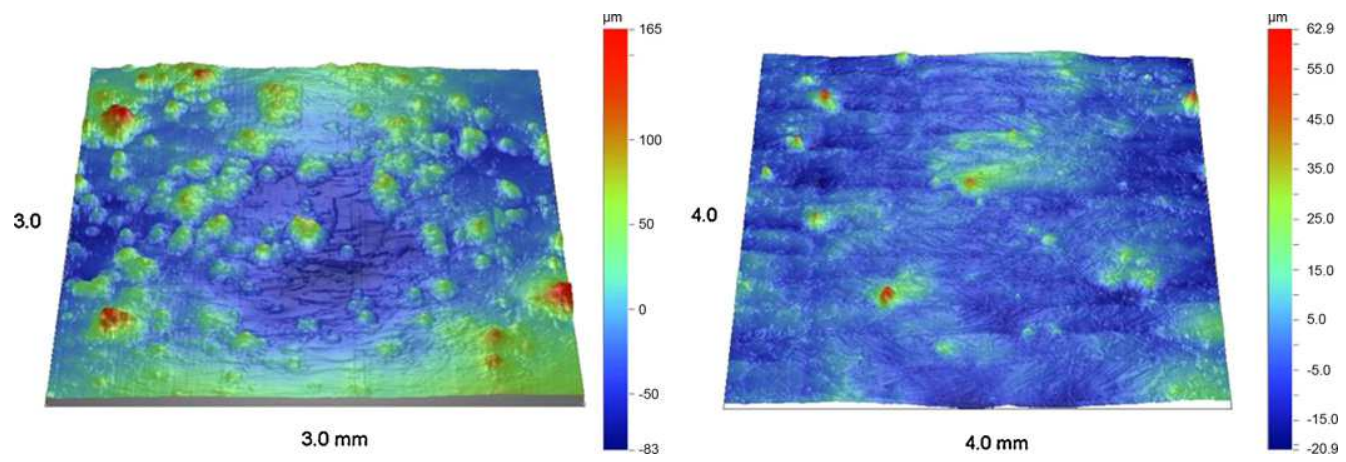

Fig. 8. 3D profiles of DMD surfaces $\left(D_{\mathrm{m}}=1 \mathrm{~g} / \mathrm{min}, d_{\mathrm{i}}=1 \mathrm{~mm}\right)$ : (a) $P=400 \mathrm{~W}, V=0.2 \mathrm{~m} / \mathrm{min}$, (b) $P=500 \mathrm{~W}, V=0.4 \mathrm{~m} / \mathrm{min}$ : agglomerates are less pronounced when $P / V$ increases.

(a)

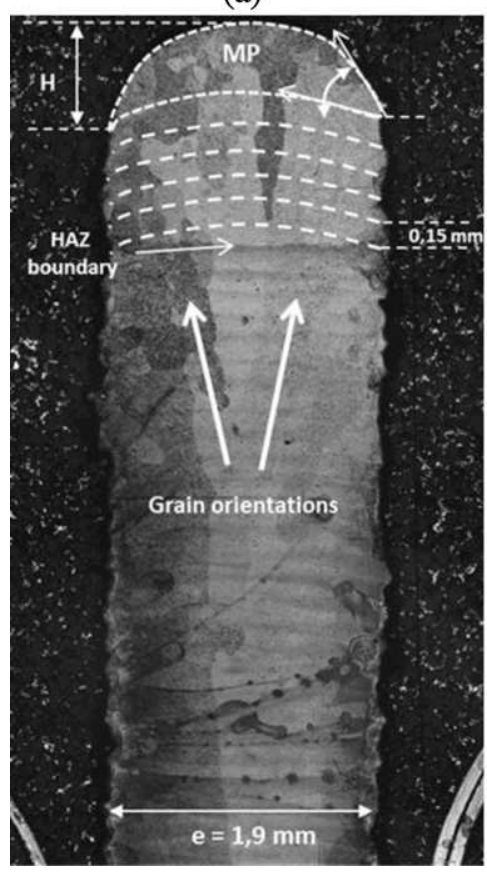

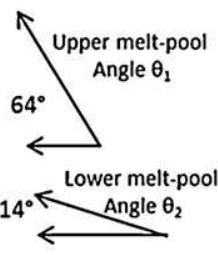

(b)

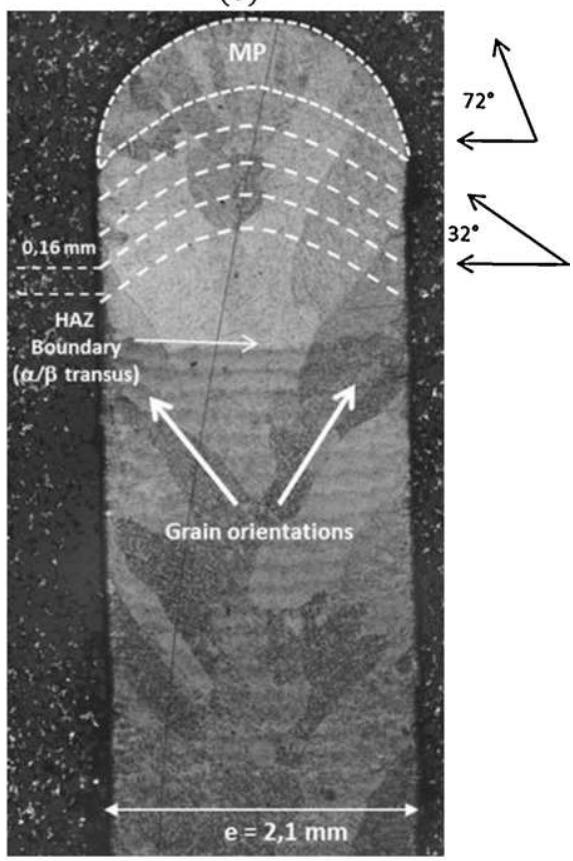

Fig. 9. Cross sections of Ti-6Al-4V walls $\left(D_{\mathrm{m}}=1 \mathrm{~g} / \mathrm{min}, V=0.4 \mathrm{~m} / \mathrm{min}\right)$ after Kroll's etching - (a) $P_{0}=320 \mathrm{~W}$, (b) $P_{0}=500 \mathrm{~W}$. The lateral menisci are smoothed by the use of a higher laser power $P_{0}$, and the melt pools lower curvature angle $\theta_{2}$ increases with high $P / V$ lineic energy $(\mathrm{J} / \mathrm{m})$ ratios, due to negative Marangoni flow that pushes upwards the central part of the melt-pool. 


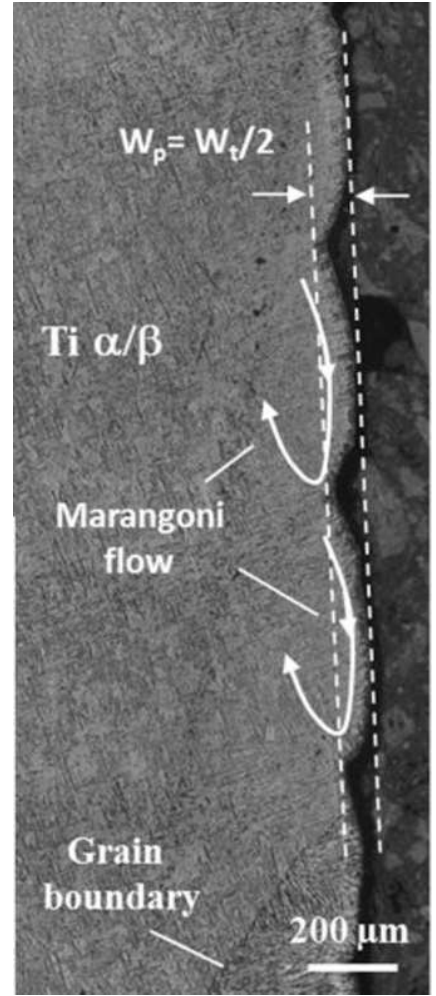

Fig. 10. Periodic meniscus formation on a Ti-6Al-4V wall $\left(0.2 \mathrm{~m} / \mathrm{min}, D_{\mathrm{m}}=1 \mathrm{~g} / \mathrm{min}\right.$, $500 \mathrm{~W}), W_{\mathrm{t}}=120 \mu \mathrm{m}-$ Expected localization of Marangoni thermo-capillary flow.

gradients $d T / d x$ and an activation of Marangoni flow (downwards at the edges of the wall, upwards in the center). Another direct effect of the Marangoni flow, is the modification of grain orientations (Fig. 9): a higher concavity of the melt-pool reduces longitudinal grain growths, and favors corolla-like but still-columnar grains structure.

For the specific case of a $0.1 \mathrm{~m} / \mathrm{min}$ scan speed, thick layers are obtained (near $\Delta h=1 \mathrm{~mm}$ ) and, for moderate laser powers ( $320 \mathrm{~W}$ on Fig. 9), the dilution factor between layers is limited. The increase in laser power (320-500 W) results in deeper and wider melt-pools, higher dilution factors $(1-\Delta h / H)$, and better surface finishes.

Macroscopic $(W)$ and microscopic $(R)$ surface finishes were reported (Fig. 11) as a function of process parameters such as: laser power $P_{0}(\mathrm{~W})$, scanning speed $V(\mathrm{~m} / \mathrm{s})$ and interaction distance $d_{\mathrm{i}}$ $(\mathrm{mm})$. For a constant average mass feed rate $D_{\mathrm{m}}$, results indicate that:

(1) High scanning speeds promote small waviness parameters $W_{t}$ (Fig. 11b), and small menisci (the mean meniscus height is approximately equal to $W_{\mathrm{t}} / 2$ : Fig. 10 ), but have a rather limited effect on micro-roughness data (Fig. 11a);

(2) Long interaction distances, corresponding to powder particles that have been molten during their time-of-flight, promote low $R_{\mathrm{t}}$ roughness, but do not modify $W_{\mathrm{t}}$ values (Fig. 11 );

(3) High laser powers tend to reduce both waviness and roughness parameters.

Consequently, the best surface parameters were obtained for high scanning speeds and high laser powers. This corresponds to thin and hot liquid layers (high scanning speeds promote short melt-pools and small $\Delta h$ values, as predicted by Eq. (7)).

The specific behavior of projected particles is mostly responsible for the micro-roughness topography:
(1) First, the melting of particles during their time-of-flight (with high power and long interaction distance) allows them to splat and to expand laterally when impacting the solid wall near the melt-pool, resulting in a reduced contribution to micro-roughness. On the other hand, non-melted solid particles agglomerate on the wall surface with a more or less pronounced necking effect, and contribute to the surface roughening;

(2) Second, high $P / V$ ratios (high $P$, low $V$ ) that promote larger meltpools, increase the catch efficiency ratio $\vartheta$ (Fig. 5), and limit the number of particles that impact the solid part of the wall and contribute to roughening. This is particularly obvious for short interaction distances where particles are kept at a solid state before reaching the melt-pool (Fig. 11a);

(3) Third, a number of particles has been shown (by CCD fast camera) to be only partially melted by the melt-pool, and to form agglomerates on the wall surface. In that case, large and hot melt-pools increase the dilution rate of particles and also improve surface finish.

If we now consider the formation of periodic menisci, the meltpool behavior has to be mostly considered as a key factor instead of the particle behavior. This was made possible by using CCD camera determinations of melt-pool size and dynamics.

\subsection{Correlation between surface finish and melt-pool sizes}

To provide a better understanding to the variation of surface finish with process parameters, a correlation was attempted with melt-pool geometry, using melt-pool data from fast camera analysis (apparent melt-pool height $H$ at the lateral edge of walls: Fig. 4), and final geometries of the walls (layer height $\Delta h$, layer width $e$ ). It was found, with a rather good fitting, that meniscus height $\left(W_{t} / 2\right)$ decrease linearly with high $H / \Delta h$ ratios, and increase linearly with the shape factor of the melt-pool H/e (Fig. 12). This shows that smaller menisci, and more planar sample surfaces can be obtained with wide and deep melt-pools, and with thin additive layers $\Delta h$. This also indicates that large and deep melt-pools are more stable that smaller ones, even if gravity forces $F_{\mathrm{g}}=\rho g H$ are more pronounced. In other words, when considering a Ti-6Al-4 melt-pool, capillary forces $\left(F_{\mathrm{c}}=2 \sigma / R\right)$ coming from surface tensions $\sigma(\mathrm{N} / \mathrm{m})$ are a dominant factor versus gravity-induced melt-pool collapse. This is confirmed by the calculation of the Bond number Bo (Eq. (7)) which is the ratio of gravity pressure over surface tension pressure: for a $1 \mathrm{~mm}$ height melt-pool, and $R=1 \mathrm{~mm}$ radius, $\mathrm{Bo} \approx 0.1$.

$B o=\frac{\rho g h}{2 \sigma / R} \approx 0.1$

The real effect from larger melt-pool volumes may be ascribed to many factors such as: hotter melt-pool surfaces and lower viscosities, provoking larger Marangoni centrifugal velocities at the lateral side, and resulting in a smoothing effect. Consequently, large temperature gradients in the melt-pool, are expected to promote smoother surfaces, due to the enhanced downward liquid flow at the lateral free surfaces of the fusion zone (Fig. 10).

\subsection{Influence of a uniform laser beam distribution on surface finish}

Starting from the conclusions mentioned above, a "top-hat" laser distribution is expected to result in lower temperature gradients in the melt-pools, and lower Marangoni effects. To confirm this, additional DMD trials have been carried out using a $1.68 \mathrm{~mm}$ diameter top-hat irradiation, and similar $\left(P, V, D_{\mathrm{m}}\right)$ conditions. As shown in Section 3.1, rather low modifications of wall dimensions were demonstrated. 

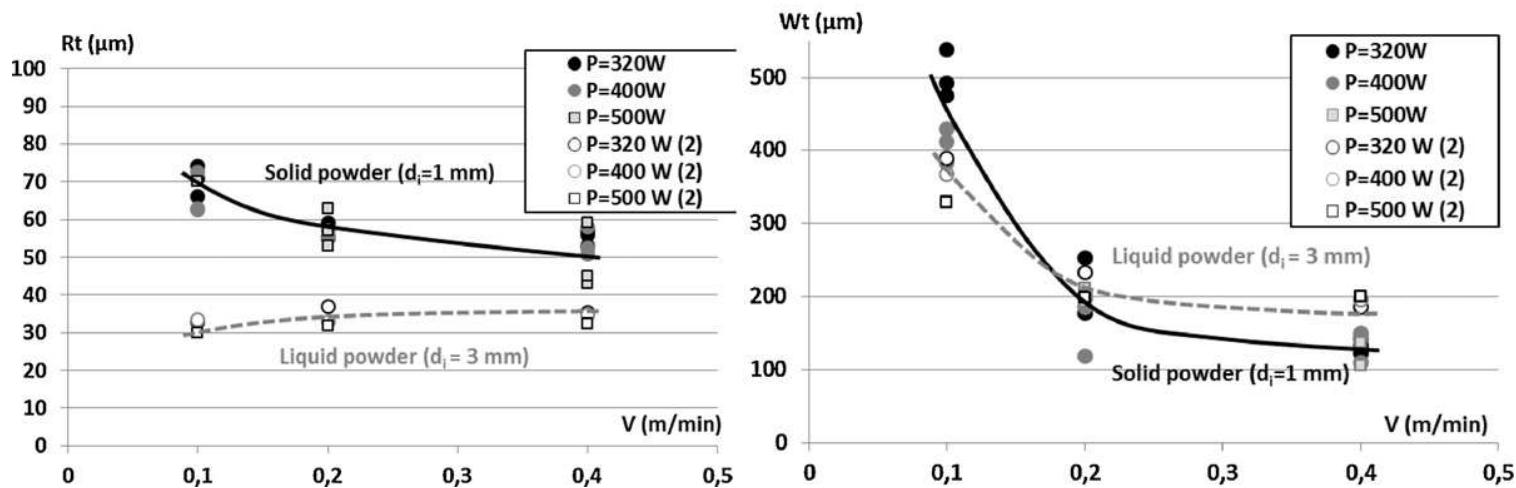

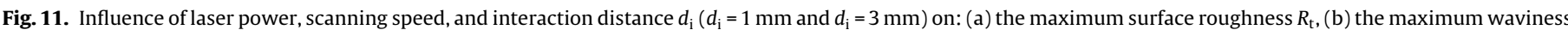
amplitude $W_{\mathrm{t}}\left(D_{\mathrm{m}}=\right.$ constant $\left.=2 \mathrm{~g} / \mathrm{min}\right)$.

Concerning surface finish, the use of a $1.68 \mathrm{~mm}$ "top-hat" distribution indicates the following tendencies:

- The optimum surface finish is still obtained with a combination of high power and low scan speeds (high $P / V$ values);

(a)

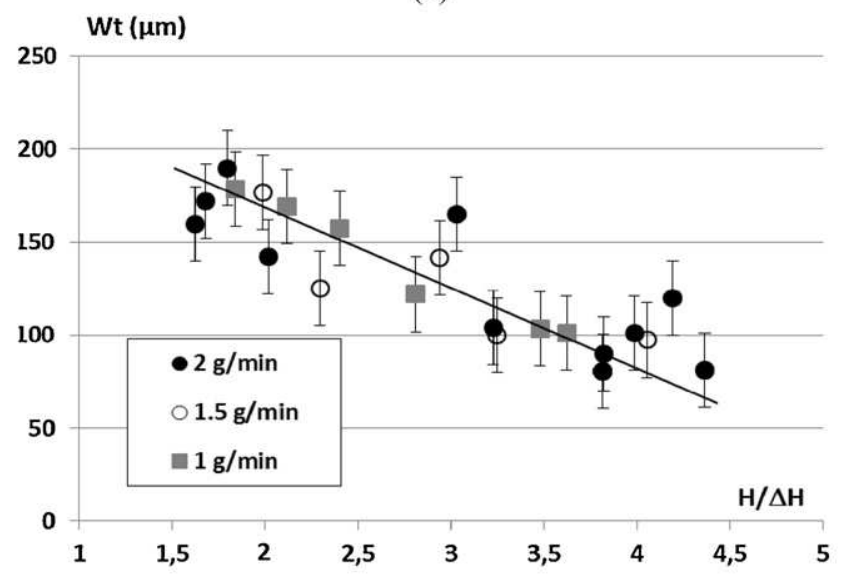

(b)

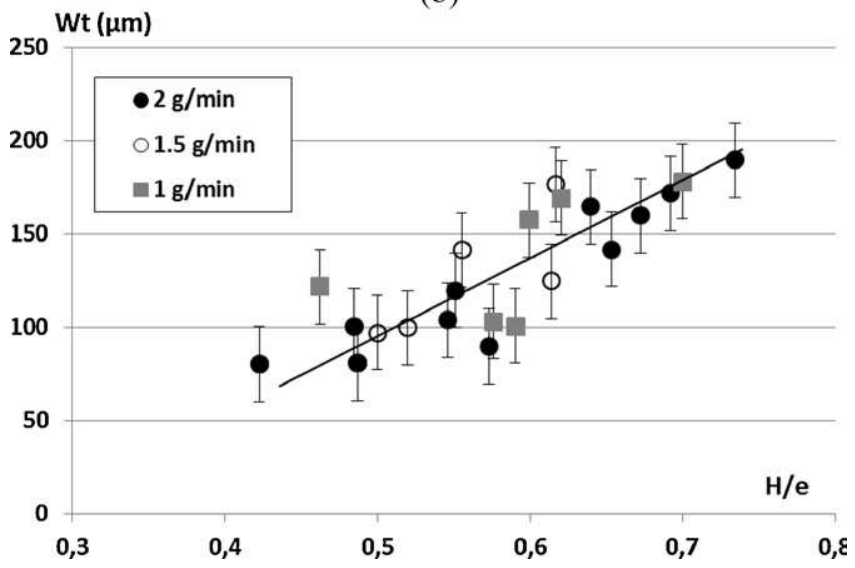

Fig. 12. (a) Influence of the $H / \Delta h$ ratio on the meniscus formation and resulting surface finish - reduced meniscus heights are obtained for high dilution ratios, (b) Influence of the $\mathrm{H} / \mathrm{e}$ aspect ratio: meniscus heights are reduced by small $\mathrm{H} / \mathrm{e}$ ratios.
- Surfaces are less undulated (factor 2 decrease of $W_{\mathrm{t}}$ and $W_{\mathrm{p}}$ values) than with a Gaussian irradiation (Fig. 13). This result seems rather contradictory with the assumption of a beneficial effect from negative Marangoni flow (Section 3.2.2) evidenced under Gaussian irradiation. A possible explanation could come from the viscosity dependence versus temperature $\eta(\mathrm{Pa} \mathrm{s})=f(T(\mathrm{~K}))$. If a large viscosity reduction occurs at high temperature, near the edges of the wall for a top-hat distribution, this should promote enhanced vertical flow, and reduce menisci amplitude. However, $\eta=f(T)$ curves are not easily available in the literature.

- The micro-roughness is not modified. This is somewhat consistent with the similar wall dimensions for Gaussian and top-hat irradiations that conduce to similar powder stream/melt-pool interactions.

\section{Analytical description of surface finish}

To confirm experimental correlations between melt-pool geometries and lateral menisci, an analytical model was developed, with an analytical description for every melt-pool, considering a similar approach than Fathi et al. (2006). On a 2D cross section, each melt-pool was considered as the sum of two semi-ellipses $x^{2} / a^{2}+y^{2} / b^{2}=1$ (one for the upper part, and one for the lower part) The analytical formulation of the upper half ellipse was validated by a comparison with the cross section of a manufactured wall (Fig. 14a). Concerning the lower half ellipse, we assumed $H_{2}=H_{1} / n$ (Fig. 14b), and a concave melt-pool/solid transition due the

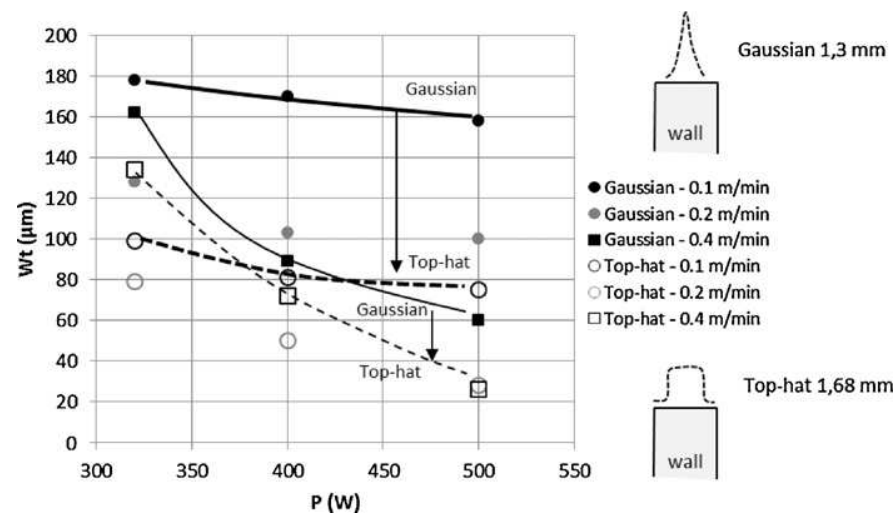

Fig. 13. Influence of a top-hat laser irradiation on maximum waviness parameter $W_{\mathrm{t}}\left(D_{\mathrm{m}}=1 \mathrm{~g} / \mathrm{min}\right)$ for three different scanning speeds $(0.1 \mathrm{~m} / \mathrm{min}, 0.2 \mathrm{~m} / \mathrm{min}$, $0.4 \mathrm{~m} / \mathrm{min})$. 

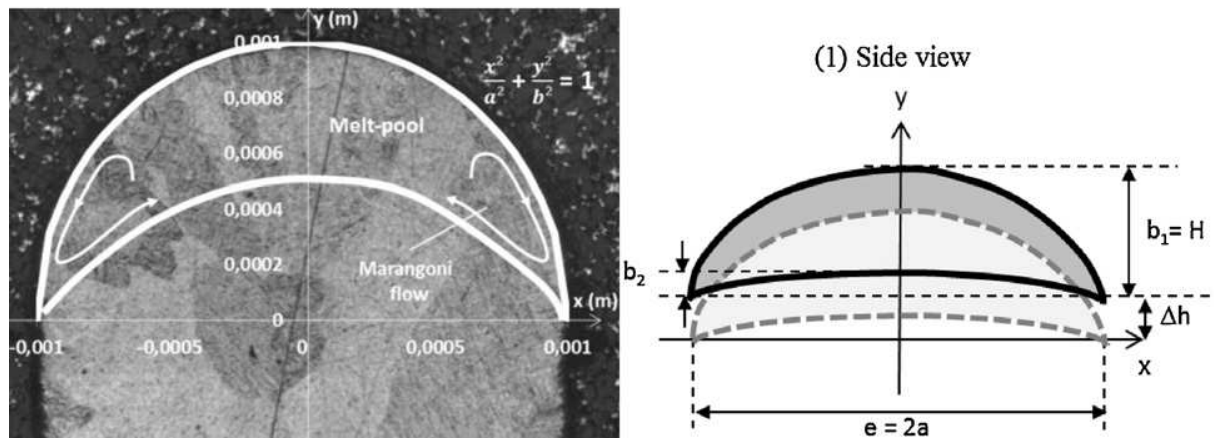

(2) Zoom on intersection zone

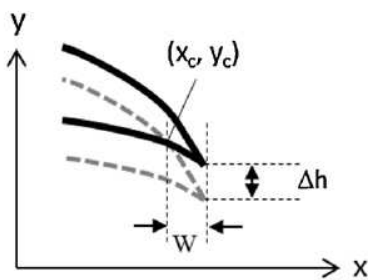

Fig. 14. Description of the analytical model - (a) identification of the ellipse equation ( $a$ " $i$ " exponent $=2$ gives a good fitting with experimental cross section considering the melt-pool shape at the top of a DMD wall, (b) basics of the model: the intersection between two half ellipses shifted of a $\Delta h$ value allows calculating $W_{\mathrm{p}}$.

negative Marangoni flow. The intersection of these two semiellipses, shifted by a $\Delta h$ value, at a $\left(x_{c}, y_{c}\right)$ location, allowed us calculating the average meniscus height $W_{\mathrm{p}}\left(\approx W_{\mathrm{t}} / 2\right)$. The resulting equations (Eqs. (9) and (10)) confirm that $W_{\mathrm{p}}$ increases linearly with layer width e, and also depends on $H / \Delta h$, as shown experimentally.

$$
\begin{aligned}
& W_{p}=\frac{e}{2}-X_{c}=\frac{e}{2}\left(1-\left(\sqrt{1-\left(\frac{\Delta h}{H_{1}(1-(1 / n))}\right)^{2}}\right)\right) \\
& \text { for } \frac{d \sigma}{d t}<0 \\
& W_{\mathrm{p}}=\frac{e}{2}-X_{\mathrm{c}}=\frac{e}{2}\left(1-\left(\sqrt{1-\left(\frac{\Delta h}{H_{1}(1+(1 / n))}\right)^{2}}\right)\right) \\
& \text { for } \frac{d \sigma}{d t}>0
\end{aligned}
$$

One of the greatest limitations of this simple model comes from the a priori definition of the melt-pool geometry, and the unmodified melt-pool shape when process parameters change. The only adjustable factor is the $\left(n=H_{1} / H_{2}\right)$ shape factor (Fig. 14b), mostly dependent on thermo-convective flow and on the $\gamma_{\mathrm{T}}=d \sigma / d T$ coefficient, which is kept constant. Considering cross-sections, it was shown that $H_{2}$ was very small compared with $H_{1}$. For the numerical application of the model, we considered $n=4$, which provides the best fitting with experimental data. The model reproduces correctly the evolution of waviness versus $H / \Delta h$ (Fig. 15) except for $H / \Delta h<2$ where the lower part of the melt-pool tends to be flatter and is not properly reproduced by well-established negative thermo-capillary flow. In the near future, a better analytical formulation of the melt-pool geometry, taking into account realistic $\theta$ angles and their evolution versus $\left(P, V, D_{\mathrm{m}}\right)$ parameters, would certainly improve the model. As a comparison, the same calculation was also carried out considering a positive Marangoni flow (Eq. (9)), and a convex melt-pool shape. In that case, the agreement is better with experimental data at low $H / \Delta h$ values because lower melt-pool curvature becomes less pronounced and $\mathrm{n}$ factor increases. Moreover, the elliptic shape assumption is not fully true for the lower melt-pool limit, especially near the lateral edges. This partially explains the difference observed in Fig. 15 between experiments and analytical predictions of $W$ values.

Current 2D thermo-hydraulic calculations of the DMD process, and oncoming temperature measurements in the melt-pool using multi-wavelength pyrometer already used by Gharbi et al. (2012) will also help us understanding the specific influence of materials' properties $\left(\sigma(\mathrm{N} / \mathrm{m}), \eta(\right.$ Pa s $\left.), \rho\left(\mathrm{kg} / \mathrm{m}^{3}\right)\right)$, and temperature gradients near the laser-powder-melt-pool interaction zone. (a)

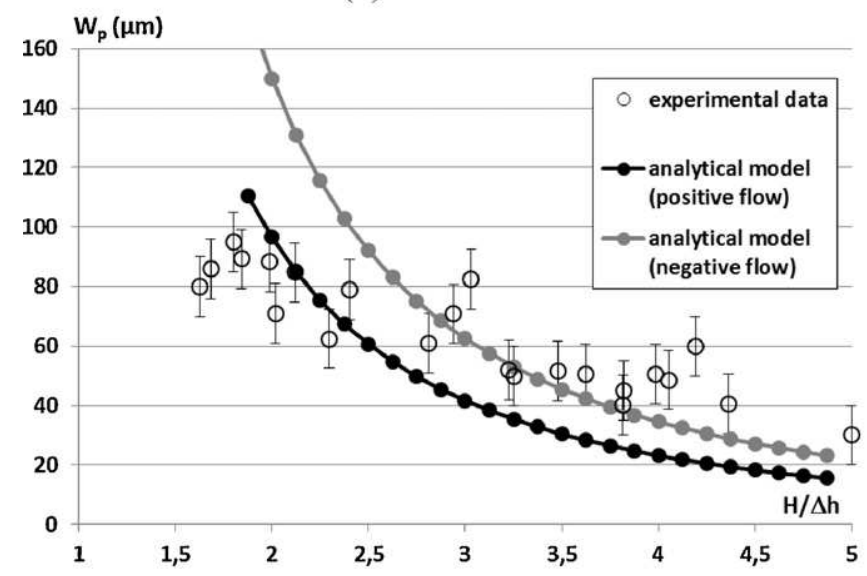

(b)
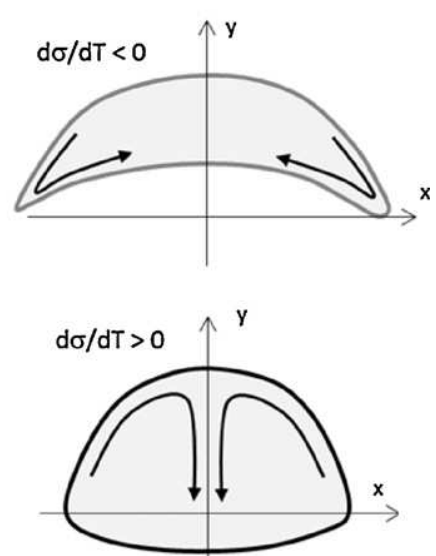

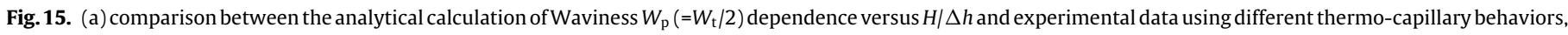
(b) scheme of negative and positive thermo-capillary flows for a central and Gaussian laser irradiation, inducing severe transverse thermal gradients. 


\section{Conclusions}

In this work, a specific focus has been put on surface finish issues, which are one of the main limitations of the DMD process. A distinction has been proposed between micro-roughness and macro-waviness parameters, as two specific contributors to surface modifications. It has been shown that the use of thin additive layers, and large melt-pools improve surface finish, and that increased powder/laser interaction distances, resulting in particle melting are also beneficial factors for surface finish. Last, a correlation has been established experimentally and validated analytically between periodic meniscus height, and melt-pool geometry. The confrontation of these data with recent thermohydraulic calculations will allow us, in the near future, improving the prediction of surface finish versus process parameters, and materials' properties.

\section{Acknowledgements}

The authors would like to acknowledge the financial support of the French National Agency for Research (ANR) under the frame of the ASPECT Project (ANR- 09-BLAN-0014).

\section{References}

Alimardani, M., Fallah, V., Iravani-Tabrizipour, M., Khajepour, A., 2012. Surface finish in laser solid freeform fabrication of an AISI 303L stainless steel thin wall. Journal of Materials Processing Technology 212,113-119.

Brandl, E., Palm, F. Michailov, V., Viehweger, B., Leyens, C., 2011 “Mechanical properties of additive manufacturing titanium (Ti-6Al-4V) blocks deposited by a solid-state laser and wire". Materials \& Design 32.
Bontha, S. The effect of process variables on microstructures in laser-deposited materials), PhD Thesis, Wright State University (USA), 2006.

Fachinetti, V.D., Cardona, A., Cosimo, A., Baufeld, B., Van Der Biest, O., 2010. "Evolution of temperature during shaped metal deposition: finite element predictions versus observations". Mecánica Computacional Vol XXIX 4915-4926 $15-18$.

Fathi, A., Toyserkani, E., Khajepour, A., Durali, M., 2006. Prediction of melt pool depth and dilution in laser powder deposition. Journal of Physics D: Applied Physics 39, 2613-2623.

Gharbi, M., Peyre, P., Fabbro, R., et al.,2012. Etude thermique des bains liquides et influence sur l'état de surface des pièces fabriquées par le procédé de fabrication directe par projection laser (FDPL). In: Conférence SFT. Bordeaux, France.

Kumar, A., Roy, S., 2009. "Effect of three-dimensional melt pool convection on process characteristics during laser cladding". Computation Materials Science 46, 495.

Maisonneuve, J., Colin, C., Aubry, P., 2007. "Profil project: direct manufacturing of aerospace components by laser cladding and laser sintering". In: Icaleo'2007 Conference, Oct 2007, Scottsdale (USA).

Morville, S., Carin, M., Carron, D., Le Masson, P., Peyre, P., Gharbi, M., Gorny, C., Fabbro R., 2011. "2D finite element modeling of heat transfer and fluid flow during mult layered DMD laser process". In: ICALEO'2011 International Conference, Orlando (USA), 17-21 Oct 2011.

Peyre, P., Neveu, R., Aubry, P., Fabbro, R., Longuet, A., 2008. “Analytical and numerica modelling of the direct metal deposition laser process". Journal of Physics D Applied Physics 41, 025403.

Pinkerton, A.J., Li, L., 2003. "The effect of laser pulse width on multiple-layer 316L steel clad microstructure and surface finish". Applied Surface Science 208-209, 405-410.

Pinkerton, A.J., 2010. "Laser direct metal deposition: theory and applications in manufacturing and maintenance". In: Lawrence, J., Pou, J., Low, D.K., Toyserkani, E. (Eds.), Advances in Laser Materials Processing. Woodhead Publishing Limited.

Qi, H., Mazumder, J., Ki, H., 2006. Numerical simulation of heat transfer and fluid flow in coaxial laser cladding process for direct metal deposition". Journal of Applied Physics 100 (2).

Zhu, G., Li, D., Zhang, A., Pi, G., Tang, Y., 2012. The influence of laser and powder defocusing characteristics on the surface quality in laser direct metal deposition. Optics \& Laser Technology 44, 349-356. 